\title{
In Situ TEM Observation on the Growth and Agglomeration of Propylene Carbonate-based Electrolytes During Sodiation with Graphene Liquid Cell
}

\author{
Joon Ha Chang ${ }^{1,2}$, Jun Young Cheong ${ }^{2}$, Il-Doo Kim $^{2}$ and Jeong Yong Lee ${ }^{1,2}$ \\ 1. Center for Nanomaterials and Chemical Reactions, Institute for Basic Science (IBS), Daejeon, \\ Korea (305-701) \\ 2. Department of Materials Science and Engineering, Korea Advanced Institute of Science and \\ Technology, Daejeon, Korea (305-701)
}

Since the first publication in Science in 2012 [1], graphene liquid cell (GLC) has attracted considerable attention with subsequent research works as it can be easily fabricated and can be used for observation in conventional transmission electron microscopy (TEM) without further modification. As nanoscience is significantly progressing each year, there is increasing demand for understanding more detailed processes of how growth and reorganization of nanoparticles or nanocrystals occur in the liquid solution.

Nevertheless, so far, little research has taken place on the growth dynamics or even transport dynamics of gel-like substances or organic polymers. Among them, not much effort has yet undertaken to understand how the electrolytes in the battery are decomposed and how decomposed electrolytes behave during the charge and discharge process. Here we have investigated the behaviour of propylene carbonate (PC) -based electrolytes during the sodiation process using GLC on a real time scale. To make GLC, monolayer graphene was first fabricated by chemical vapor deposition (CVD) method and electrolyte (in this case, PC-based electrolyte with active material) was encapsulated between two graphene sheets to be loaded on TEM. Upon e-beam irradiation, electrolytes are reduced and undergo decomposition process, where we can readily observe many different shapes of decomposed electrolytes moving across different areas.

The actual vial glass containing the PC-based electrolytes and active material to be used for this experiment is shown in Figure 1. Time-series TEM observation of how the decomposed electrolytes appear and behave during the sodiation process is specifically depicted in Figure 2. It is interesting to note that the decomposed electrolytes actually appear and that these decomposed electrolytes actually undergo growth and agglomeration, which are somewhat similar to the nanoparticle growth processes shown in previous literatures [2,3,4]. At $80 \mathrm{~s}$, decomposed electrolytes start to appear in the snapshot, having small sizes. These small decomposed electrolytes either merge with one another (120 s or $184 \mathrm{~s}$ ) or grow into larger decomposed electrolytes. The decomposed electrolytes grow larger $(240 \mathrm{~s})$ and they agglomerate into even larger electrolytes (300 s and $321 \mathrm{~s})$. As can be seen, the growth and agglomeration of decomposed electrolytes occur simultaneously, where the size of the decomposed electrolytes increases as sodiation process proceeds.

In summary, we have demonstrated, through time-series TEM observation, the growth and agglomeration of decomposed electrolytes during sodiation. Using GLC to understand more about the growth and agglomeration of decomposed electrolytes can open up new opportunities to learn more about the electrolyte decomposition process that is integral to the overall electrochemical processes. [5]

References:

[1] J. M. Yuk et al, Science 336 (2012), p. 61-64. 
[2] H. Zheng et al, Science 324 (2009), p. 1309-1312.

[3] H.-G. Liao et al, Science 336 (2012), p. 1011-1014.

[4] J. M. Yuk et al, ACS Nano 8 (2014), p. 7478-7485.

[5] The authors acknowledge the funding from Korea CCS R\&D Center (KCRC) grant by the Korea government (Ministry of Science, ICT \& Future Planning) (No. NRF-2014M1A8A1049303), EndRun grant from KAIST by the Korea government in 2015 (Ministry of Science, ICT \& Future Planning) (N01150615), and Institute for Basic Science (IBS) (IBS-R004-G3).

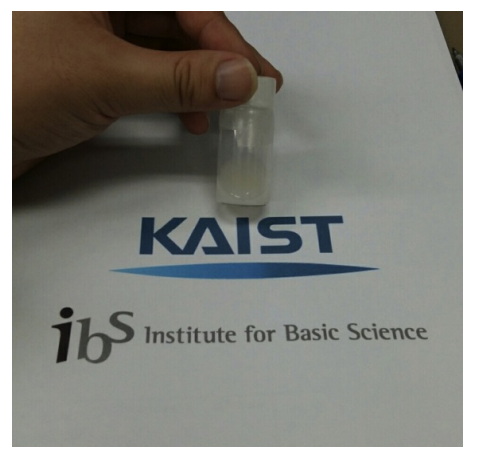

Figure 1. Digital image of vial glass containing PC-based electrolytes mixed with active material.
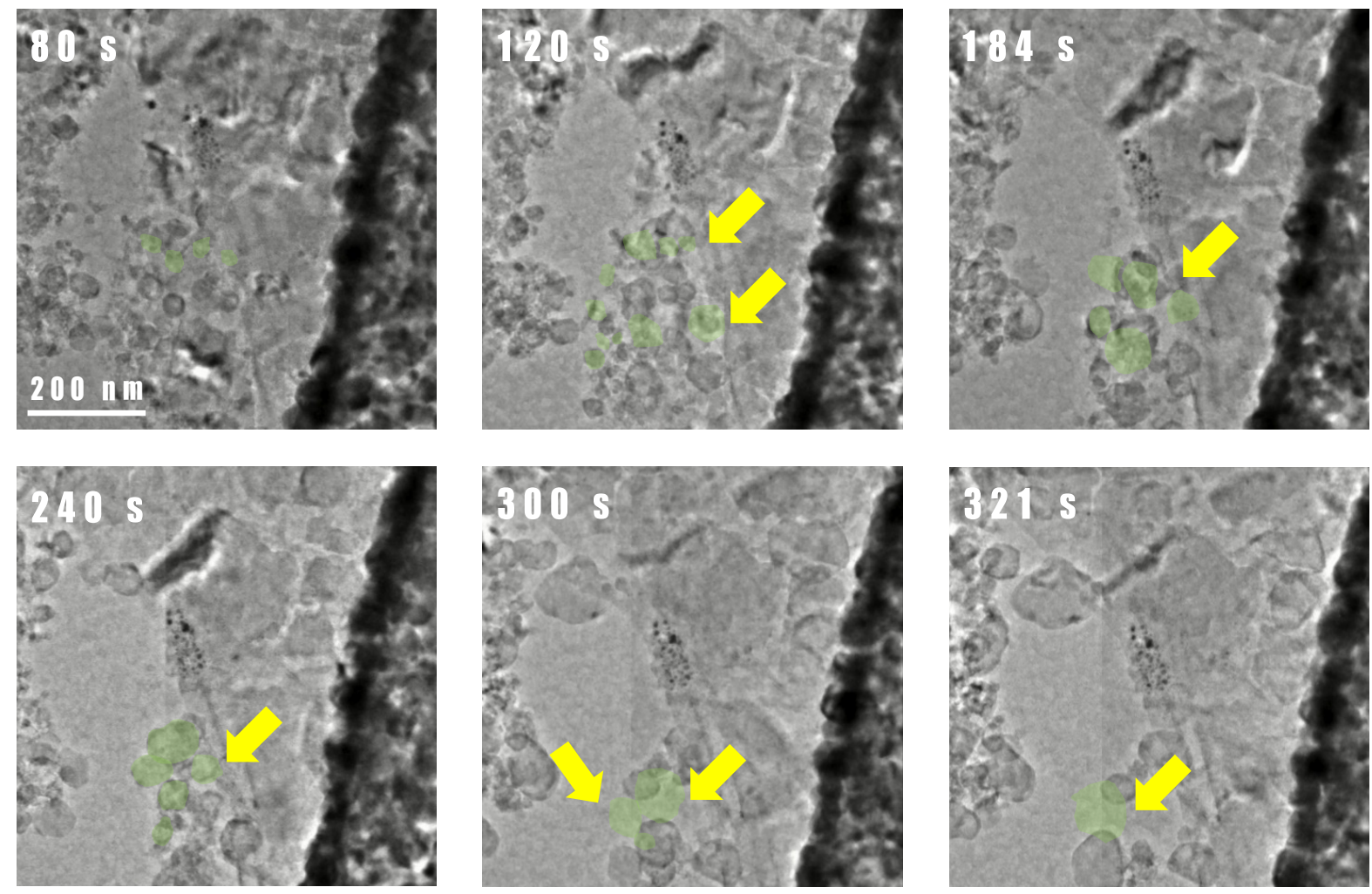

Figure 2. Time-series TEM observation images showing the growth and agglomeration of PC-based electrolytes under sodiation process. 